\title{
The Association between Fat Mass, Lean Mass and Bone Mineral Density in Premenopausal Women in Korea: A Cross-Sectional Study
}

\author{
Jeehyun Kim', Hyuktae Kwon ${ }^{1, *}$, Bo-Kyoung Heo ${ }^{1, *}$, Hee-Kyung Joh², Cheol Min Lee ${ }^{3}$, Seung-Sik Hwang ${ }^{4}$, \\ Danbee Park', Jae-Hong Park ${ }^{1}$ \\ 'Department of Family Medicine, Seoul National University Hospital, Seoul, Korea \\ ${ }^{2}$ Department of Family Medicine, Seoul National University Health Service Center, Seoul, Korea \\ ${ }^{3}$ Department of Family Medicine, Healthcare System Gangnam Center, Seoul National University Hospital, Seoul, Korea \\ ${ }^{4}$ Department of Social and Preventive Medicine, Inha University School of Medicine, Incheon, Korea
}

Background: We investigated the association between body composition, especially truncal or non-truncal fat mass (FM), and bone mineral density (BMD) in premenopausal women in Korea.

Methods: A cross-sectional study was performed using data from the Korea National Health and Nutrition Examination Survey IV and V (2008-2011). Total lean mass (LM), total FM (TFM), truncal FM, and non-truncal FM, and BMD of the total femur, femoral neck (FN), and lumbar spine were measured using dual-energy X-ray absorptiometry. The association between body composition and BMD was analyzed using multiple linear regression. The risk of low BMD according to quartiles of TFM, truncal FM, and non-truncal FM was calculated using logistic regression. Subgroup analysis according to body mass index was also performed.

Results: In 4,343 premenopausal women, total LM was positively associated with BMD regardless of weight adjustment. TFM, truncal FM, and non-truncal FM were inversely associated with BMD after adjusting for weight. Odds ratios (ORs) for low BMD and 95\% confidence intervals (CIs) of the highest quartile of TFM, truncal FM, and nontruncal FM compared with the lowest quartile were calculated. The risk of low BMD of the FN was higher in the highest quartile of TFM (OR, 4.48; 95\% CI, 1.11-18.01) and truncal FM (OR, 5.48; 95\% CI, 1.75-17.20). Truncal FM and not-truncal FM had an inverse association with BMD in the non-obese and obese subgroups of women.

Conclusion: Total LM has a protective effect on BMD and FM can have a detrimental effect on BMD besides its skeletal loading effect.

Keywords: Bone Density; Fat Mass; Lean Mass; Premenopause; Osteoporosis

Received: September 2, 2016, Revised: September 30, 2016, Accepted: October 14, 2016

*Corresponding Author: Hyuktae Kwon https://orcid.org/0000-0002-0312-3650

Tel: +82-2-2072-4039, Fax: +82-2-766-3276, E-mail: ezkel@snuh.org

*Corresponding Author: Bo-Kyoung Heo https://orcid.org/0000-0002-5326-2085

Tel: +82-2-2072-4039, Fax:+82-2-766-3276, E-mail: ultraman123@naver.com

*These authors contributed equally to this work. 


\section{INTRODUCTION}

Osteoporosis is a systemic skeletal disease characterized by low bone mass and microarchitectural deterioration of bone tissue. ${ }^{1)}$ It has become an important health issue worldwide, as its prevalence increases with advancing age. Estimates of the prevalence of osteoporosis vary according to its definition, but the World Health Organization (WHO) has estimated that $30 \%$ of all women aged over 50 years (postmenopausal) have osteoporosis according to a definition of bone mineral density (BMD) lower than 2.5 standard deviations from the mean for young healthy adult women at any site. ${ }^{2)}$

BMD is a major determinant of the risk for bone fracture, which causes serious morbidity and mortality in the elderly. ${ }^{3)}$ Among the several factors that affect bone health, body weight is one of the strongest positive predictors of bone mass. ${ }^{4)}$ It increases bone mass by increasing the mechanical stress on the skeleton, thereby increasing the stimulus for osteogenesis; ${ }^{5)}$ conversely, loss in body weight leads to loss in bone mass. However, how fat mass (FM) and lean mass (LM) - the two major components of body weight-contribute to BMD remains debatable.

Several studies have suggested a positive association between LM and BMD in women, ${ }^{6-8)}$ while FM has shown an inconsistent association with BMD. ${ }^{8-11)}$ The subjects of previous studies have mostly been postmenopausal women or the elderly; however, research on the bone health of premenopausal women is also important, since BMD starts decreasing before menopause. Large cross-sectional studies in the Korean population have also reported inconsistent results on the association between FM and BMD. ${ }^{12-14)}$ In addition to this inconsistency, the subjects in previous studies were not representative of the general Korean population.
The inconsistency in the association between FM and BMD might be attributable to the complex mechanisms of the effects of FM on $\mathrm{BMD}$. FM is believed to have a positive effect on BMD through the mechanical loading of soft tissue. Meanwhile, FM, especially visceral fat, can affect bone health through hormonal mechanisms; however, the effect of these mechanisms on BMD requires further investigation. Recent studies on the effect of fat distribution on BMD showed an inverse association between visceral fat and BMD in women. ${ }^{15,16)}$

We hypothesized that LM would be positively associated with BMD, while FM, especially truncal FM (assuming that it contains mostly visceral fat), might show a negative association with BMD after adjusting for the mechanical loading effect. In the present study, we aimed to investigate the association between FM, classified according to its distribution in the body, and BMD in premenopausal women, on the basis of the Korea National Health and Nutrition Examination Survey (KNHANES) IV and V, which represent the general Korean population.

\section{METHODS}

\section{Subjects}

Our cross-sectional study included 5,531 premenopausal women aged 20 years and above, who had undergone dual-energy X-ray absorptiometry (DXA) scans between 2008 and 2011; the study used data from the KNHANES IV and V. The KNHANES, a nationwide survey representing the health and nutritional status of the non-institutionalized civilian population of Korea, consists of health interviews, nutrition surveys, and health examinations. A stratified, multistage probability sampling design was used, and sampling units were based on the geographical area, age, and sex. All subjects provided informed consent prior to their inclusion in the study. ${ }^{17)}$

Total participants in the KNHANES IV and V $(\mathrm{N}=37,753)$

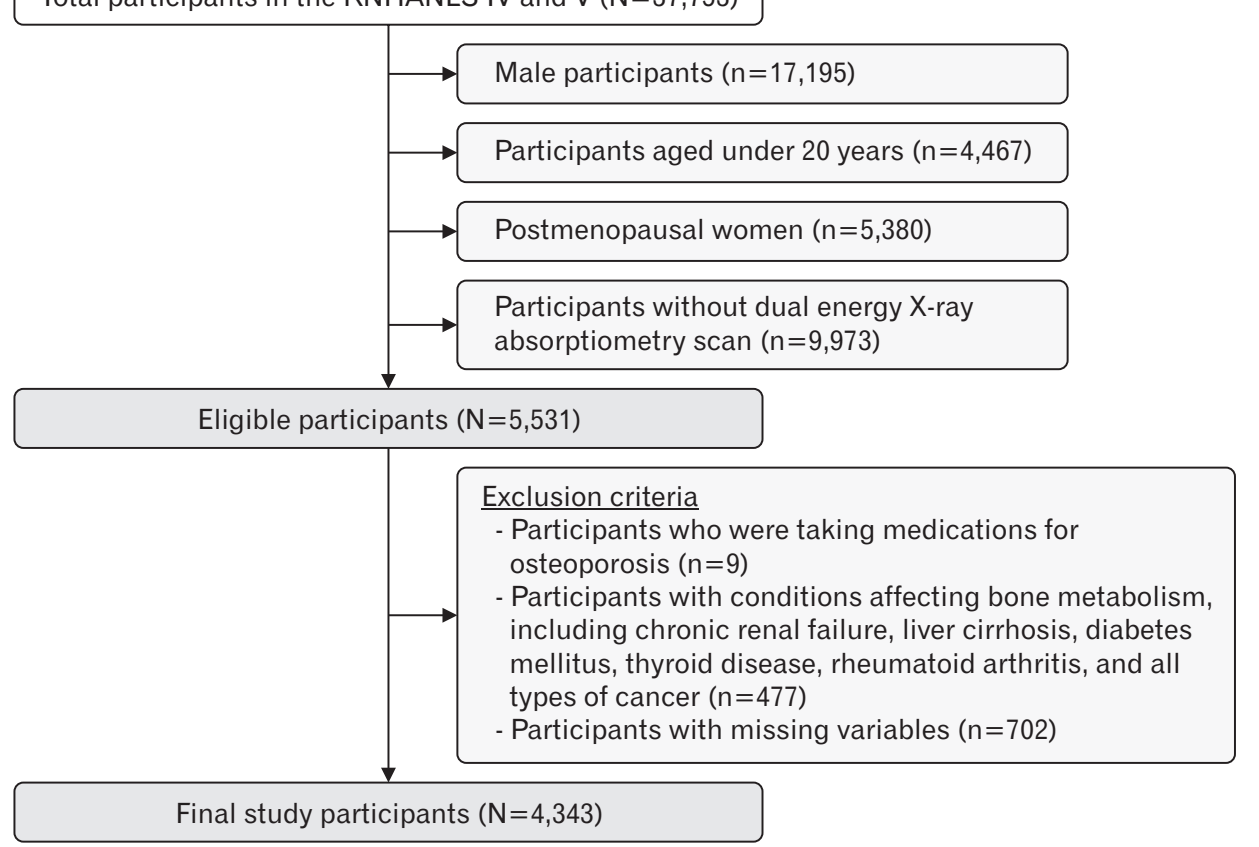

Figure 1. Study population. KNHANES, Korean National Health and Nutrition Examination Survey. 
Of the 5,531 participants, we initially excluded nine subjects who reported that they were taking medication for osteoporosis. In addition, we excluded 477 participants with conditions that might affect bone metabolism, including chronic renal failure, diabetes mellitus, liver cirrhosis, rheumatoid arthritis, thyroid disease, and all types of cancer. Among the 5,045 eligible participants, we further excluded those with missing values for any of the study variables $(n=702,13.9 \%)$. A total of 4,343 women were included in our final analysis (Figure 1). Since the KNHANES is a publicly available dataset, this study did not require ethical approval of an Institutional Review Board.

\section{Dual-Energy X-ray Absorptiometry Measurement of Body Composition, Bone Mineral Density Analysis, and Z-score}

All participants of the present study had undergone DXA (DISCOVERY-W fan-beam densitometer; Hologic Inc., Waltham, MA, USA) to assess BMD and body composition. The KNHANES IV (2008-2009) and V (2010-2011) included large-scale whole-body DXA measurements, in which trained engineers generated an accurate and reliable dataset. The results of DXA were analyzed using industry-standard techniques at the Korean Society of Osteoporosis with the Hologic Discovery software ver. 13.1 (Hologic Inc.) in its default configuration. ${ }^{17}$ )

Daily standardized quality control of DXA instruments was performed using a spine phantom provided by the manufacturer before the start of the study. ${ }^{17)}$ For body composition analysis, total FM (TFM, $\mathrm{kg}$ ), total LM (TLM, kg) excluding bone mass, and truncal FM (TrFM, $\mathrm{kg}$ ) were measured, in addition to BMD $\left(\mathrm{g} / \mathrm{cm}^{2}\right)$ of the total femur, femoral neck, and lumbar spine. Non-truncal FM (nTrFM, kg) was also calculated to represent appendicular FM, by subtracting the TrFM from the FM of the subtotal body (entire body excluding the head).

The normal range of BMD among premenopausal women was defined using the Z-scores of the BMD of the total femur, femoral neck, and lumbar spine by age, sex, and race according to International Society for Clinical Densitometry guidelines. ${ }^{18)}$ Since the KNHANES data represents the general Korean population, we calculated the means and standard deviations of BMD for each age and sex from all subjects included in the KNHANES IV and V, and used them as reference for assessing the Z-scores.

\section{Measurement of Other Study Variables}

Trained interviewers collected demographic data and information on health-related behaviors of the study participants via personal interviews. The demographic variables included age (years), highest educational level achieved (up to or less than elementary school education, middle or high school education, and college or higher education), and monthly household income (in quartiles). The equivalent household income was calculated as the total monthly household income divided by the square root of the total number of household members. ${ }^{17)}$

Information regarding health-related behavioral factors including smoking (never, past, or current smoker), alcohol consumption (non-, moderate, or heavy drinker), and physical activity (low, moderate, or high) was also obtained. Heavy drinkers were defined as subjects who consumed more than $120 \mathrm{~g}$ of alcohol per week, according to the KNHANES IV and V. ${ }^{17)}$ Physical activity was quantified as a metabolic equivalent of task minutes (MET-minutes) per week, which was calculated using the scoring protocol of the Korean version of the International Physical Activity Questionnaire short form. ${ }^{19)}$ Accordingly, physical activity levels were classified as low ( $<600$ MET-minutes per week), moderate ( $\geq 600$ to $<1,500$ MET-minutes per week), or high $(\geq 1,500$ MET minutes per week). Daily calcium intake (mg) was evaluated using the 24-hour recall method.

Blood samples were collected from each participant during the survey and were processed as follows: antecubital vein blood samples were drawn and were immediately centrifuged. Plasma total cholesterol (mg/dL), high-density lipoprotein cholesterol (mg/dL), low-density lipoprotein cholesterol $(\mathrm{mg} / \mathrm{dL})$, triglyceride $(\mathrm{mg} / \mathrm{dL})$, fasting glucose (fasting at least 12 hours, $\mathrm{mg} / \mathrm{dL}$ ), and fasting insulin concentrations $(\mu \mathrm{IU} / \mathrm{mL})$ were measured enzymatically using a Hitachi 7600 Automatic Analyzer (Hitachi, Tokyo, Japan). ${ }^{17)}$ Insulin resistance was evaluated using the homeostasis model assessment for estimated insulin resistance (HOMA-IR) index (i.e., HOMA-IR=(fasting plasma glucose $) \times\left(\right.$ fasting plasma insulin)/405) ${ }^{20)}$ Serum 25 -hydroxyvitamin D concentration $(\mathrm{ng} / \mathrm{mL}$ ) was measured by radioimmunoassay (DiaSorin Inc., Stillwater, MN, USA), using a $\gamma$-counter (1470 Wizard; PerkinElmer, Wallac, Turku, Finland). ${ }^{17}$

Anthropometric factors, including body weight and height, were obtained using standard protocols and were measured to the nearest $0.1 \mathrm{~kg}$ and $0.1 \mathrm{~cm}$, respectively. Body mass index (BMI) was calculated as body weight in kilograms divided by the squared height in meters $\left(\mathrm{kg} / \mathrm{m}^{2}\right)$.

\section{Statistical Analysis}

To analyze the combined survey samples over 4 years, average sampling weights were used to generate unbiased cross-sectional estimates representative of the general Korean population. The characteristics of the study participants were presented as means \pm standard errors for continuous variables and percentages for categorical variables. Multiple linear regression analysis was performed to assess the association between body composition (TFM, TLM, TrFM, nTrFM) and BMD of the total femur, femoral neck, and lumbar spine after adjusting for confounding variables including age, height, education level, monthly income, daily calcium intake, serum 25-hydroxyvitamin D concentration, and health-related behaviors including smoking, physical activity, and alcohol consumption (model 1). To independently evaluate the association between body composition and BMD from the possible confounding effects of body weight, multiple regression analyses were performed after additional adjustment for body weight in model 1 (model 2). In addition, to evaluate whether insulin resistance or serum cholesterol levels mediated the association between TFM and BMD, HOMA-IR and serum lipid profiles were separately added as covariates to model 2 , and the results of analyses with and without these covariates were compared. 
Further, we divided participants into "non-obese" (BMI $<25 \mathrm{~kg} / \mathrm{m}^{2}$ ) and "obese" (BMI $\geq 25 \mathrm{~kg} / \mathrm{m}^{2}$ ) subgroups, and performed multiple linear regression analysis in both subgroups separately as well as after adjusting for BMI in order to verify the association between TrFM, nTrFM, and BMD according to BMI. The cutoff value of BMI for obesity was based on WHO recommendations for Asian populations. ${ }^{21)}$ In addition, we performed multivariate logistic regression analyses to estimate the odds ratios (ORs) and 95\% confidence intervals (CIs) for low BMD (Z-score <-2.0) by quartiles of TFM, TrFM, and nTrFM, using the lowest quartile as reference and adjusting for all variables included in the multiple regression model 2.

All statistical analyses were performed using STATA ver. 14.1 (Stata Corp., College Station, TX, USA), and P-values $<0.05$ were considered statistically significant.

\section{RESULTS}

The baseline characteristics of the study participants (mean age, 35.6

Table 1. Baseline characteristics of study participants according to Z-scores

\begin{tabular}{|c|c|c|c|}
\hline Characteristic & Z-score $\geq-2.0(n=4,218)$ & Z-score $<-2.0(n=125)$ & P-value \\
\hline Age (y) & $35.6 \pm 0.2$ & $35.2 \pm 0.8$ & 0.62 \\
\hline Height (cm) & $159.6 \pm 0.1$ & $156.6 \pm 0.6$ & $<0.01$ \\
\hline Weight (kg) & $57.6 \pm 0.2$ & $48.4 \pm 0.6$ & $<0.01$ \\
\hline Body mass index $\left(\mathrm{kg} / \mathrm{m}^{2}\right)$ & $22.6 \pm 0.1$ & $19.7 \pm 0.2$ & $<0.01$ \\
\hline Education level (\%) & & & 0.25 \\
\hline$\leq$ Elementary school & 4.3 & 2.1 & \\
\hline Middle/high school & 53.5 & 57.8 & \\
\hline$\geq$ College & 42.2 & 40.1 & \\
\hline Household income (\%) & & & 0.14 \\
\hline Q1 (low) & 8.3 & 14.9 & \\
\hline Q2 & 27.0 & 25.9 & \\
\hline Q3 & 32.7 & 30.8 & \\
\hline Q4 (high) & 32.0 & 28.4 & \\
\hline Smoking status (\%) & & & 0.72 \\
\hline Never & 84.9 & 86.2 & \\
\hline Past & 8.7 & 6.4 & \\
\hline Current & 6.4 & 7.4 & \\
\hline Alcohol consumption (\%) & & & $<0.01$ \\
\hline No & 49.5 & 63.5 & \\
\hline Moderate & 43.0 & 34.0 & \\
\hline Heavy* & 7.5 & 2.5 & \\
\hline Physical activity ${ }^{\dagger}(\%)$ & & & 0.75 \\
\hline Low & 75.4 & 75.2 & \\
\hline Moderate & 21.0 & 22.3 & \\
\hline High & 3.6 & 2.5 & \\
\hline Daily calcium intake (mg) & $465.2 \pm 6.0$ & $475.5 \pm 28.3$ & 0.72 \\
\hline Vitamin D (ng/mL) & $16.0 \pm 0.2$ & $14.1 \pm 0.6$ & $<0.01$ \\
\hline Total cholesterol (mg/dL) & $177.9 \pm 0.6$ & $173.1 \pm 2.7$ & 0.08 \\
\hline Triglyceride (mg/dL) & $93.1 \pm 1.2$ & $87.5 \pm 6.0$ & 0.36 \\
\hline High-density lipoprotein cholesterol (mg/dL) & $57.2 \pm 0.2$ & $58.2 \pm 1.1$ & 0.37 \\
\hline Low-density lipoprotein cholesterol (mg/dL) & $104.9 \pm 1.1$ & $96.8 \pm 5.0$ & 0.11 \\
\hline Homeostasis model assessment-estimated insulin resistance & $2.2 \pm 0.2$ & $1.9 \pm 0.9$ & $<0.01$ \\
\hline Body composition $(\mathrm{kg})$ & & & $<0.01$ \\
\hline Total lean mass & $36.4 \pm 0.1$ & $32.0 \pm 0.4$ & \\
\hline Total fat mass & $18.6 \pm 0.1$ & $14.3 \pm 0.4$ & \\
\hline Truncal fat mass & $8.9 \pm 0.1$ & $6.3 \pm 0.2$ & \\
\hline Non-truncal fat mass & $8.8 \pm 0.1$ & $7.2 \pm 0.2$ & \\
\hline Bone mineral density $\left(\mathrm{g} / \mathrm{cm}^{2}\right)$ & & & $<0.01$ \\
\hline Total femur & $0.904 \pm 0.002$ & $0.699 \pm 0.007$ & \\
\hline Femoral neck & $0.769 \pm 0.002$ & $0.580 \pm 0.006$ & \\
\hline Lumbar spine & $0.991 \pm 0.002$ & $0.771 \pm 0.008$ & \\
\hline
\end{tabular}

Values are presented as mean \pm standard error or $\%$. P-values $<0.05$ were presented in bold text. Sampling weights were applied to statistical analysis for unbiased crosssectional estimates. P-values were calculated using t-test for continuous variables and $\chi^{2}$ test for categorical variables.

MET, metabolic equivalent of task.

*Heavy drinking was defined as consumption of more than $120 \mathrm{~g}$ of alcohol per week. 'Physical activity level was classified as low (<600 MET-min/wk), moderate ( $\geq 600$ to $<1,500 \mathrm{MET}-\mathrm{min} / \mathrm{wk})$, and high ( $\geq 1,500 \mathrm{MET}-\mathrm{min} / \mathrm{wk})$. 
years) included in the final analysis are shown in Appendix 1. Table 1 compares the characteristics of the two groups of participants divided according to their Z-scores. Overall, approximately $2.9 \%$ of the participants had Z-scores below -2.0 ( $\mathrm{n}=125)$. Participants with Z-scores more than or equal to -2.0 were taller, heavier, and drank less alcohol than those with Z-scores below -2.0, and had higher serum 25-hydroxyvitamin D concentration, HOMA-IR level, and higher TLM, TFM, TrFM, and nTrFM than the corresponding values of participants with Z-scores below $-2.0(\mathrm{P}<0.05)$.

Table 2 shows the multivariate-adjusted associations between body composition (TFM, TLM, TrFM, and nTrFM) and BMD of the total femur, femoral neck, and lumbar spine. TLM was positively associated with BMD regardless of adjustment for weight. In contrast, although TFM, TrFM, and nTrFM were positively associated with BMD in model 1 , the association became negative after adjusting for body weight (model 2). In addition, although the gradient of the abovementioned analysis changed after adjusting for HOMA-IR or cholesterol level, neither the direction of the association between TFM and BMD nor the statistical significance of the analysis changed (Appendix 2).

We also compared the association between TrFM, nTrFM, and BMD according to BMI subgroup, by multivariate-adjusted linear regression analysis (Appendix 3). Both TrFM and nTrFM showed an overall negative association with BMD in non-obese and obese participants, after adjusting for body weight.

No significant differences in the risk for low BMD (Z-score <-2.0) were observed across quartiles of TFM, TrFM, and nTrFM after adjusting for potential confounders including body weight. With regard to the parts of the body from which the BMD was measured, the risk of low BMD at the femoral neck was significantly higher in the highest quartile of TFM and TrFM compared with that in the lowest quartile. The ORs for low BMD of the femoral neck were 4.48 (95\% CI, 1.1118.01) for TFM, 5.48 (95\% CI, 1.75-17.20) for TrFM, and 3.27 (95\% CI,
0.49-21.98) for nTrFM (Figure 2).

\section{DISCUSSION}

Our study demonstrated that in premenopausal Korean women, TLM was positively associated with BMD regardless of body weight, while TFM was inversely associated with BMD after adjusting for body weight. However, both TrFM and nTrFM, which were assumed to represent visceral and subcutaneous fat, respectively, were inversely associated with BMD.

Body weight is one of the strongest positive predictors of bone mass, ${ }^{4)}$ and previous studies have suggested that LM is an important determinant of bone density in women, ${ }^{6-8)}$ including premenopausal women. ${ }^{8,22)}$ In concordance with previous studies, our study found that LM is positively associated with BMD in premenopausal Korean women. Since this relationship remained even after adjusting for body weight, we concluded that LM affects bone mas through mechanisms other than its direct loading effect on the skeleton via muscle contractions that result from physical activity. ${ }^{6)}$ Genetic, environmental, and hormonal components are also involved in these mechanisms. ${ }^{23-25)}$

FM, on the other hand, was variably associated with BMD after adjusting for body weight. TFM, TrFM, and nTrFM were positively associated with BMD without adjusting for body weight, but were all negatively associated with BMD after adjusting for body weight, consistent with previous studies performed among the Korean population. ${ }^{12-14,26,27)}$

A recent meta-analysis has shown that both LM and FM are positively associated with BMD across all ages and ethnicities. ${ }^{28)}$ Most studies that reported positive associations between FM and BMD assessed the association between body composition and BMD without adjusting for body weight. ${ }^{8,9,11,12)}$ Since LM and FM comprise most of the body weight and are considered to work together to maintain bone

Table 2. Association between body composition (TLM, TFM, TrFM, nTrFM) and BMD

\begin{tabular}{|c|c|c|c|c|c|c|}
\hline \multirow{3}{*}{ Variable } & \multicolumn{6}{|c|}{ Bone mineral density $\left(\mathrm{g} / \mathrm{cm}^{2}\right)$} \\
\hline & \multicolumn{2}{|c|}{ Total femur } & \multicolumn{2}{|c|}{ Femoral neck } & \multicolumn{2}{|c|}{ Lumbar spine } \\
\hline & $\beta \pm S E$ & P-value & $\beta \pm S E$ & P-value & $\beta \pm S E$ & P-value \\
\hline \multicolumn{7}{|l|}{ Model $1^{*}$} \\
\hline TLM $(\mathrm{kg})$ & $0.1181 \pm 0.0005$ & $<0.001$ & $0.0096 \pm 0.0005$ & $<0.001$ & $0.0098 \pm 0.0005$ & $<0.001$ \\
\hline TFM $(\mathrm{kg})$ & $0.0069 \pm 0.0003$ & $<0.001$ & $0.0060 \pm 0.0003$ & $<0.001$ & $0.0061 \pm 0.0004$ & $<0.001$ \\
\hline $\operatorname{TrFM}(\mathrm{kg})$ & $0.1115 \pm 0.0005$ & $<0.001$ & $0.0094 \pm 0.0005$ & $<0.001$ & $0.0100 \pm 0.0006$ & $<0.001$ \\
\hline$n \operatorname{TrFM}(\mathrm{kg})$ & $0.0144 \pm 0.0009$ & $<0.001$ & $0.0129 \pm 0.0008$ & $<0.001$ & $0.0125 \pm 0.0009$ & $<0.001$ \\
\hline \multicolumn{7}{|l|}{ Model $2^{\dagger}$} \\
\hline TLM $(\mathrm{kg})$ & $0.0052 \pm 0.0009$ & $<0.001$ & $0.0030 \pm 0.0010$ & 0.002 & $0.0029 \pm 0.0009$ & 0.001 \\
\hline TFM (kg) & $-0.0074 \pm 0.0009$ & $<0.001$ & $-0.0055 \pm 0.0009$ & $<0.001$ & $-0.0058 \pm 0.0008$ & $<0.001$ \\
\hline $\operatorname{TrFM}(\mathrm{kg})$ & $-0.0092 \pm 0.0013$ & $<0.001$ & $-0.0075 \pm 0.0014$ & $<0.001$ & $-0.0065 \pm 0.0014$ & $<0.001$ \\
\hline $\mathrm{nTrFM}(\mathrm{kg})$ & $-0.0095 \pm 0.0014$ & $<0.001$ & $-0.0057 \pm 0.0014$ & $<0.001$ & $-0.0084 \pm 0.0014$ & $<0.001$ \\
\hline
\end{tabular}

Sampling weights were applied to statistical analysis for unbiased cross-sectional estimates. $\beta$, SE, and P-values were calculated using multiple linear regression. P-values $<0.05$ are presented in bold text.

TLM, total lean mass; TFM, total fat mass; TrFM, truncal fat mass; nTrFM, non-truncal fat mass; $\beta, \beta$-coefficient; SE, standard error.

${ }^{*}$ Adjusted for age, height, smoking status, alcohol consumption, physical activity, household income, education, daily calcium intake, and serum vitamin $D$ concentration.

${ }^{\dagger}$ Adjusted for weight in addition to all variables in model 1. 
A

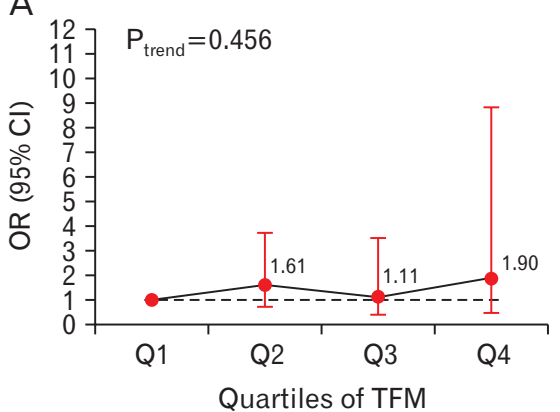

B

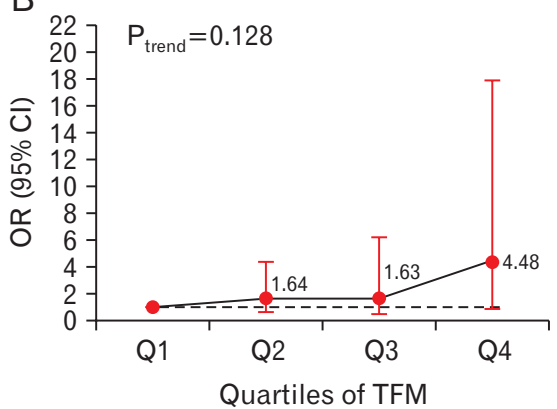

C

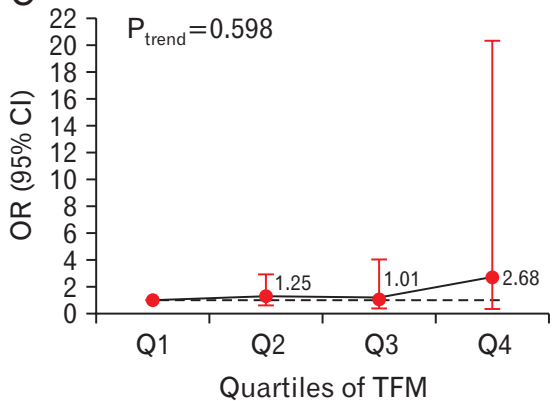

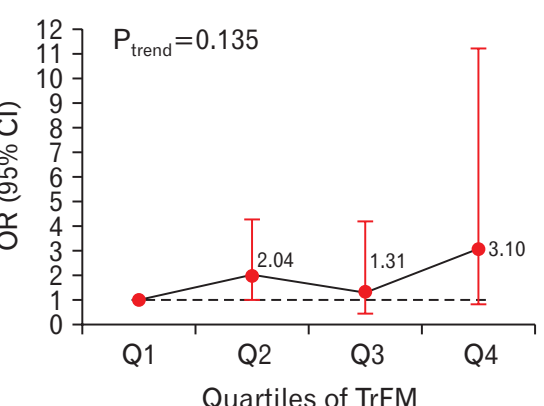
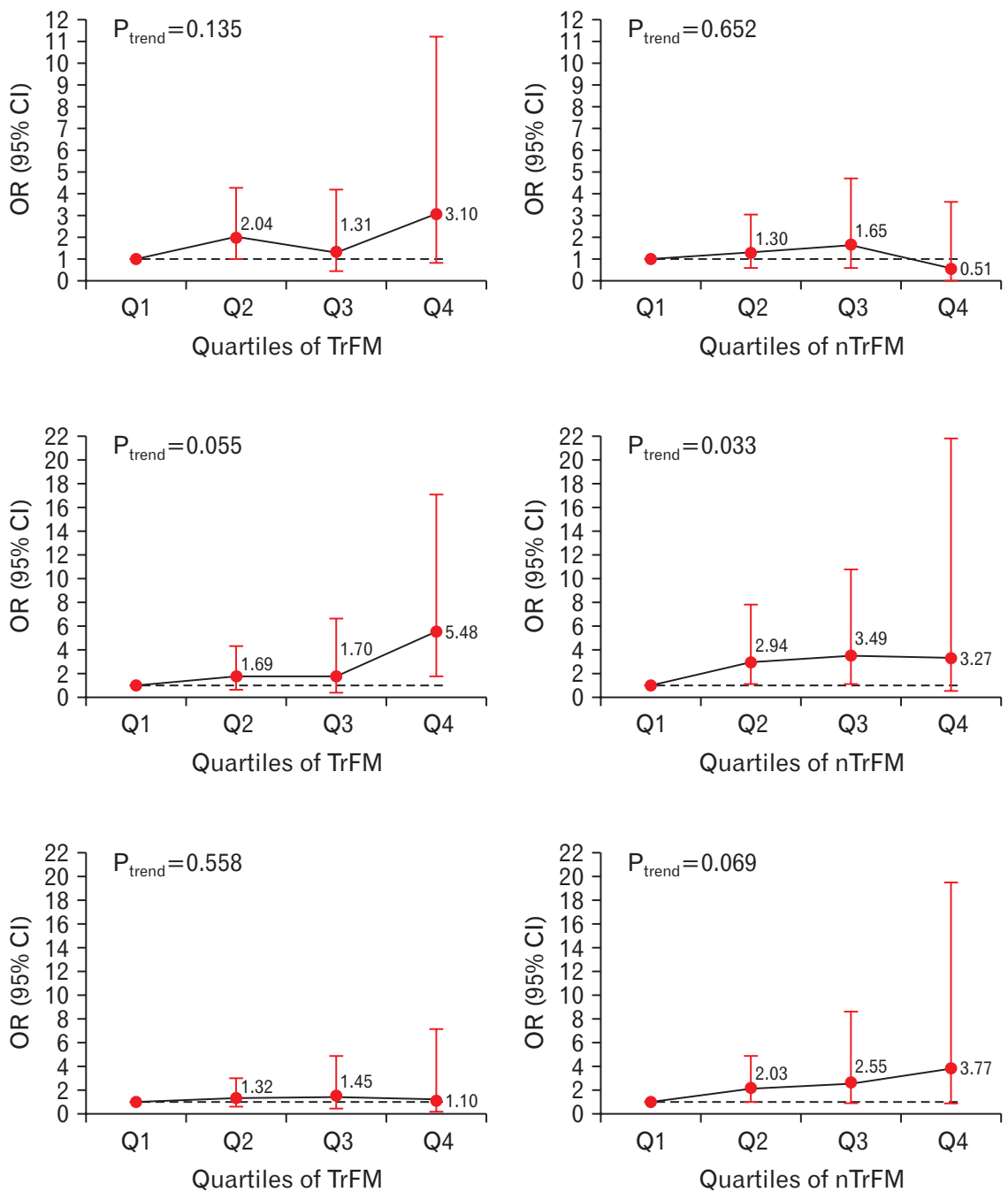

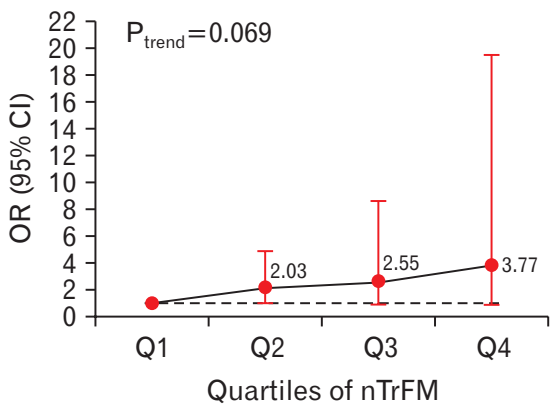

Figure 2. Risk of low BMD (Z-score $<-2.0$ ) across quartiles of TFM, TrFM, and nTrFM. (A) BMD of the total femur. (B) BMD of the femoral neck. (C) Lumbar spine BMD. ORs $(95 \% \mathrm{Cl})$ were calculated using multivariate logistic regression after adjusting for age, weight, height, smoking status, alcohol consumption, physical activity, household income, education, calcium intake, and serum vitamin D concentration. BMD, bone mineral density; TFM, total fat mass; TrFM, truncal fat mass; nTrFM, non-truncal fat mass; $\mathrm{OR}$, odds ratio; $\mathrm{Cl}$, confidence interval.

mass via skeletal loading effects, collinearity likely occurs with body weight, which contributes to the inconsistent findings of previous studies. ${ }^{29)}$ Although it remained controversial with regard to statistical analysis, findings from the study suggest that FM itself might have a negative effect on BMD independent of its effect on body weight, based on the observation that compared with LM, FM had a much smaller effect on BMD without adjusting for the body weight of FM, but showed the opposite result after adjusting for body weight.

The detrimental effect of FM on BMD may be attributed to several complex mechanisms of FM and types of fat tissue. Recent studies have demonstrated the differential impact of specific fat compartments on BMD, with visceral adipose tissue having detrimental effects on BMD. ${ }^{15,16)}$ Fat and bone are linked by a multitude of pathways, and adiponectin, insulin, amylin, preptin, leptin, and adipocytic estrogens are all likely to be involved in this relationship. ${ }^{29)}$ Since insulin resis- tance or lipid profiles, which are related to obesity, mediate the association between FM and bone density owing to their effects on bone mass, ${ }^{11,29,30)}$ we included HOMA-IR and serum cholesterol level as covariates in our analysis. However, in our study, the association between FM and BMD did not change after adjusting for HOMA-IR or serum cholesterol levels, a finding that suggests that FM could also affect BMD through mechanisms other than insulin resistance or cholesterol metabolism.

In this study, we investigated the association between body composition and BMD in healthy premenopausal women from the KNHANES database, which represents the health and nutritional status of the general Korean population. To our knowledge, this is the largest cross-sectional study on this topic. Our study defined Z-scores, and the diagnostic criteria for low BMD for premenopausal women by age and sex; these results may be helpful in clinical settings. We also 
investigated whether the effect of FM on BMD differs according to fat distribution, using TrFM and nTrFM to represent visceral fat and subcutaneous fat, respectively, since direct measurement of visceral fat was not available in the KNHANES data. Contrary to our predictions, both TrFM and nTrFM were negatively associated with BMD after adjusting for body weight. Because only a small number of participants were obese in our population $(n=866)$ and all participants were premenopausal, both TrFM and nTrFM might mainly consist of subcutaneous fat, suggesting that even subcutaneous fat (besides visceral fat) can have a detrimental effect on bone health.

Our study has several limitations. First, the cross-sectional study design limits our ability to determine the causal relationship between body composition and BMD. Second, information bias cannot be ruled out, as demographic and health-related behavioral parameters were obtained from self-reported questionnaires. Third, our study results cannot be extended to other sexes, ages, and ethnicities, as the study population and cutoff values for the study variables were specific to premenopausal Korean women. Fourth, hidden confounding factors may also affect the association between body composition and BMD.

In conclusion, the findings of our study suggest that increased LM has protective effects on bone health, while increased FM may have a detrimental effect on BMD in addition to its positive weight-bearing effect, in premenopausal Korean women. Therefore, an adequate composition of LM and FM might be important for maintaining bone health and preventing osteoporosis. Further investigation is necessary to determine whether BMD differs according to the type of fat tissue based on direct measurement of visceral fat.

\section{CONFLICT OF INTEREST}

No potential conflict of interest relevant to this article was reported.

\section{REFERENCES}

1. Rizzoli R, Bonjour JP, Ferrari SL. Osteoporosis, genetics and hormones. J Mol Endocrinol 2001;26:79-94.

2. WHO Study Group. Assessment of fracture risk and its application to screening for postmenopausal osteoporosis: report of a WHO Study Group. World Health Organ Tech Rep Ser 1994;843:1-129.

3. Johnell O, Kanis JA. An estimate of the worldwide prevalence and disability associated with osteoporotic fractures. Osteoporos Int 2006;17:1726-33.

4. Felson DT, Zhang Y, Hannan MT, Anderson JJ. Effects of weight and body mass index on bone mineral density in men and women: the Framingham study. J Bone Miner Res 1993;8:567-73.

5. Beck TJ, Oreskovic TL, Stone KL, Ruff CB, Ensrud K, Nevitt MC, et al. Structural adaptation to changing skeletal load in the progression toward hip fragility: the study of osteoporotic fractures. J Bone Miner Res 2001;16:1108-19.

6. Taaffe DR, Cauley JA, Danielson M, Nevitt MC, Lang TF, Bauer DC, et al. Race and sex effects on the association between muscle strength, soft tissue, and bone mineral density in healthy elders: the Health, Aging, and Body Composition Study. J Bone Miner Res 2001;16:1343-52.

7. Pluijm SM, Visser M, Smit JH, Popp-Snijders C, Roos JC, Lips P. Determinants of bone mineral density in older men and women: body composition as mediator. J Bone Miner Res 2001;16:2142-51.

8. Wang MC, Bachrach LK, Van Loan M, Hudes M, Flegal KM, Crawford $\mathrm{PB}$. The relative contributions of lean tissue mass and fat mass to bone density in young women. Bone 2005;37:474-81.

9. Reid IR, Plank LD, Evans MC. Fat mass is an important determinant of whole body bone density in premenopausal women but not in men. J Clin Endocrinol Metab 1992;75:779-82.

10. Zhao LJ, Liu YJ, Liu PY, Hamilton J, Recker RR, Deng HW. Relationship of obesity with osteoporosis. J Clin Endocrinol Metab 2007;92:1640-6.

11. Hsu YH, Venners SA, Terwedow HA, Feng Y, Niu T, Li Z, et al. Relation of body composition, fat mass, and serum lipids to osteoporotic fractures and bone mineral density in Chinese men and women. Am J Clin Nutr 2006;83:146-54.

12. Park JH, Song YM, Sung J, Lee K, Kim YS, Kim T, et al. The association between fat and lean mass and bone mineral density: the Healthy Twin Study. Bone 2012;50:1006-11.

13. Kim JH, Choi HJ, Kim MJ, Shin CS, Cho NH. Fat mass is negatively associated with bone mineral content in Koreans. Osteoporos Int 2012;23:2009-16.

14. Kim CJ, Oh KW, Rhee EJ, Kim KH, Jo SK, Jung CH, et al. Relationship between body composition and bone mineral density (BMD) in perimenopausal Korean women. Clin Endocrinol (Oxf) 2009;71:18-26.

15. Fu X, Ma X, Lu H, He W, Wang Z, Zhu S. Associations of fat mass and fat distribution with bone mineral density in pre- and postmenopausal Chinese women. Osteoporos Int 2011;22:113-9.

16. Bredella MA, Torriani M, Ghomi RH, Thomas BJ, Brick DJ, Gerweck $\mathrm{AV}$, et al. Determinants of bone mineral density in obese premenopausal women. Bone 2011;48:748-54.

17. Korea Centers for Disease Control and Prevention. The fourth and the fifth Korea National Health and Nutrition Examination Survey (KNHANES IV \& V). Cheongju: Korea Centers for Disease Control and Prevention; 2008-2011.

18. Schousboe JT, Shepherd JA, Bilezikian JP, Baim S. Executive summary of the 2013 International Society for Clinical Densitometry Position Development Conference on bone densitometry. J Clin Densitom 2013;16:455-66.

19. Oh JY, Yang YJ, Kim BS, Kang JH. Validity and reliability of Korean version of International Physical Activity Questionnaire (IPAQ) short form. J Korean Acad Fam Med 2007;28:532-41.

20. Matthews DR, Hosker JP, Rudenski AS, Naylor BA, Treacher DF, Turner RC. Homeostasis model assessment: insulin resistance and beta-cell function from fasting plasma glucose and insulin concentrations in man. Diabetologia 1985;28:412-9.

21. World Health Organization. The Asia-Pacific perspective: redefining obesity and its treatment. Geneva: World Health Organization; 2000.

22. Lu LJ, Nayeem F, Anderson KE, Grady JJ, Nagamani M. Lean body mass, not estrogen or progesterone, predicts peak bone mineral density in premenopausal women. J Nutr 2009;139:250-6.

23. Seeman E, Hopper JL, Young NR, Formica C, Goss P, Tsalamandris C. Do genetic factors explain associations between muscle strength, lean mass, and bone density?: a twin study. Am J Physiol 1996;270(2 Pt 1): 
E320-7.

24. Nguyen TV, Howard GM, Kelly PJ, Eisman JA. Bone mass, lean mass, and fat mass: same genes or same environments? Am J Epidemiol 1998;147:3-16.

25. Lang TF. The bone-muscle relationship in men and women. J Osteoporos 2011;2011:702735.

26. Ahn SH, Lee SH, Kim H, Kim BJ, Koh JM. Different relationships between body compositions and bone mineral density according to gender and age in Korean populations (KNHANES 2008-2010). J Clin Endocrinol Metab 2014;99:3811-20.

27. Kim YM, Kim SH, Kim S, Yoo JS, Choe EY, Won YJ. Variations in fat mass contribution to bone mineral density by gender, age, and body mass index: the Korea National Health and Nutrition Examination Survey (KNHANES) 2008-2011. Osteoporos Int 2016;27:2543-54.

28. Ho-Pham LT, Nguyen UD, Nguyen TV. Association between lean mass, fat mass, and bone mineral density: a meta-analysis. J Clin Endocrinol Metab 2014;99:30-8.

29. Reid IR. Fat and bone. Arch Biochem Biophys 2010;503:20-7.

30. Lim JS, Kim KM, Rhee Y, Lim SK. Gender-dependent skeletal effects of vitamin D deficiency in a younger generation. J Clin Endocrinol Metab 2012;97:1995-2004. 
Appendix 1. Baseline characteristics of study participants $(n=4,343)$

\begin{tabular}{|c|c|}
\hline Characteristic & Value \\
\hline Age (y) & $35.6 \pm 0.2$ \\
\hline Height (cm) & $159.5 \pm 0.1$ \\
\hline Weight (kg) & $57.3 \pm 0.2$ \\
\hline Body mass index $\left(\mathrm{kg} / \mathrm{m}^{2}\right)$ & $22.5 \pm 0.1$ \\
\hline \multicolumn{2}{|l|}{ Education level (\%) } \\
\hline SElementary school & 4.3 \\
\hline Middle/high school & 53.6 \\
\hline$\geq$ College & 42.1 \\
\hline \multicolumn{2}{|l|}{ Household income (\%) } \\
\hline$Q_{1}$ (low) & 8.5 \\
\hline$Q_{2}$ & 27.0 \\
\hline$Q_{3}$ & 32.7 \\
\hline$Q_{4}$ (high) & 31.8 \\
\hline \multicolumn{2}{|l|}{ Smoking status (\%) } \\
\hline Never & 84.9 \\
\hline Past & 8.7 \\
\hline Current & 6.4 \\
\hline \multicolumn{2}{|l|}{ Alcohol consumption (\%) } \\
\hline No & 49.9 \\
\hline Moderate & 42.8 \\
\hline Heavy $^{\star}$ & 7.3 \\
\hline \multicolumn{2}{|l|}{ Physical activity ${ }^{\dagger}(\%)$} \\
\hline Low & 75.4 \\
\hline Moderate & 21.1 \\
\hline High & 3.5 \\
\hline Daily calcium intake (mg) & $465.5 \pm 5.9$ \\
\hline Vitamin D (ng/mL) & $16.0 \pm 0.2$ \\
\hline Total cholesterol (mg/dL) & $177.8 \pm 0.6$ \\
\hline Triglyceride (mg/dL) & $92.9 \pm 1.2$ \\
\hline High-density lipoprotein cholesterol (mg/dL) & $57.2 \pm 0.2$ \\
\hline Low-density lipoprotein cholesterol (mg/dL) & $104.6 \pm 1.1$ \\
\hline Homeostasis model assessment-estimated insulin resistance & $2.2 \pm 0.2$ \\
\hline \multicolumn{2}{|l|}{ Body composition (kg) } \\
\hline Total lean mass & $36.2 \pm 0.1$ \\
\hline Total fat mass & $18.4 \pm 0.1$ \\
\hline Truncal fat mass & $8.8 \pm 0.1$ \\
\hline Non-truncal fat mass & $8.8 \pm 0.1$ \\
\hline \multicolumn{2}{|l|}{ Bone mineral density $\left(\mathrm{g} / \mathrm{cm}^{2}\right)$} \\
\hline Femur total & $0.898 \pm 0.002$ \\
\hline Femur neck & $0.764 \pm 0.002$ \\
\hline Lumbar spine & $0.984 \pm 0.002$ \\
\hline
\end{tabular}

Values are presented as mean \pm standard error or \%. Sampling weights were applied to statistical analysis for unbiased cross-sectional estimates.

MET, metabolic equivalent of task.

*Heavy drinking was defined as consumption of more than $120 \mathrm{~g}$ of alcohol per week. 'Physical activity level was classified as low ( $<600 \mathrm{MET}-\mathrm{min} / \mathrm{wk})$, moderate ( $\geq 600$ to $<1,500 \mathrm{MET}-\mathrm{min} / \mathrm{wk}$ ), and high ( $\geq 1,500 \mathrm{MET}-\mathrm{min} / \mathrm{wk})$. 
Appendix 2. Association between body composition (TLM, TFM, TrFM, nTrFM) and BMD according to adjustment for HOMA-IR level and serum lipid profiles

\begin{tabular}{|c|c|c|c|c|c|c|}
\hline \multirow{3}{*}{ Variable } & \multicolumn{6}{|c|}{$\mathrm{BMD}\left(\mathrm{g} / \mathrm{cm}^{2}\right)$} \\
\hline & \multicolumn{2}{|c|}{ Total femur } & \multicolumn{2}{|c|}{ Femoral neck } & \multicolumn{2}{|c|}{ Lumbar spine } \\
\hline & $\beta \pm \mathrm{SE}$ & P-value & $\beta \pm \mathrm{SE}$ & P-value & $\beta \pm \mathrm{SE}$ & P-value \\
\hline \multicolumn{7}{|l|}{ Model 2* } \\
\hline TLM & $0.0052 \pm 0.0009$ & $<0.001$ & $0.0030 \pm 0.0010$ & 0.002 & $0.0029 \pm 0.0009$ & 0.001 \\
\hline TFM & $-0.0074 \pm 0.0009$ & $<0.001$ & $-0.0055 \pm 0.0009$ & $<0.001$ & $-0.0058 \pm 0.0008$ & $<0.001$ \\
\hline TrFM & $-0.0092 \pm 0.0013$ & $<0.001$ & $-0.0075 \pm 0.0014$ & $<0.001$ & $-0.0065 \pm 0.0014$ & $<0.001$ \\
\hline$n \operatorname{TrFM}$ & $-0.0095 \pm 0.0014$ & $<0.001$ & $-0.0057 \pm 0.0014$ & $<0.001$ & $-0.0084 \pm 0.0014$ & $<0.001$ \\
\hline \multicolumn{7}{|l|}{ Model $2-1^{\dagger}$} \\
\hline TLM & $0.0051 \pm 0.0010$ & $<0.001$ & $0.0030 \pm 0.0010$ & 0.004 & $0.0025 \pm 0.0009$ & 0.007 \\
\hline TFM & $-0.0071 \pm 0.0009$ & $<0.001$ & $-0.0052 \pm 0.0010$ & $<0.001$ & $-0.0055 \pm 0.0009$ & $<0.001$ \\
\hline TrFM & $-0.0085 \pm 0.0014$ & $<0.001$ & $-0.0068 \pm 0.0015$ & $<0.001$ & $-0.0055 \pm 0.0014$ & $<0.001$ \\
\hline$n T r F M$ & $-0.0102 \pm 0.0015$ & $<0.001$ & $-0.0066 \pm 0.0015$ & $<0.001$ & $-0.0093 \pm 0.0015$ & $<0.001$ \\
\hline \multicolumn{7}{|l|}{ Model 2-2 } \\
\hline TLM & $0.0050 \pm 0.0009$ & $<0.001$ & $0.0029 \pm 0.0010$ & 0.002 & $0.0026 \pm 0.0009$ & 0.003 \\
\hline TFM & $-0.0073 \pm 0.0009$ & $<0.001$ & $-0.0053 \pm 0.0009$ & $<0.001$ & $-0.0056 \pm 0.0009$ & $<0.001$ \\
\hline TrFM & $-0.0090 \pm 0.0013$ & $<0.001$ & $-0.0074 \pm 0.0014$ & $<0.001$ & $-0.0061 \pm 0.0014$ & $<0.001$ \\
\hline$n T r F M$ & $-0.0094 \pm 0.0014$ & $<0.001$ & $-0.0057 \pm 0.0014$ & $<0.001$ & $-0.0082 \pm 0.0014$ & $<0.001$ \\
\hline \multicolumn{7}{|l|}{ Model 2-3§ } \\
\hline TLM & $0.0052 \pm 0.0009$ & $<0.001$ & $0.0030 \pm 0.0010$ & 0.002 & $0.0029 \pm 0.0009$ & 0.001 \\
\hline TFM & $-0.0073 \pm 0.0009$ & $<0.001$ & $-0.0053 \pm 0.0009$ & $<0.001$ & $-0.0058 \pm 0.0009$ & $<0.001$ \\
\hline TrFM & $-0.0091 \pm 0.0013$ & $<0.001$ & $-0.0073 \pm 0.0014$ & $<0.001$ & $-0.0065 \pm 0.0014$ & $<0.001$ \\
\hline$n T r F M$ & $-0.0099 \pm 0.0014$ & $<0.001$ & $-0.0062 \pm 0.0014$ & $<0.001$ & $-0.0086 \pm 0.0014$ & $<0.001$ \\
\hline \multicolumn{7}{|l|}{ Model 2-4॥ } \\
\hline TLM & $0.0022 \pm 0.0015$ & 0.150 & $0.0007 \pm 0.0016$ & 0.643 & $0.0007 \pm 0.0017$ & 0.672 \\
\hline TFM & $-0.0050 \pm 0.0015$ & 0.001 & $-0.0036 \pm 0.0015$ & 0.018 & $-0.0047 \pm 0.0016$ & 0.004 \\
\hline TrFM & $-0.0036 \pm 0.0023$ & 0.118 & $-0.0030 \pm 0.0025$ & 0.219 & $-0.0038 \pm 0.0030$ & 0.171 \\
\hline$n T r F M$ & $-0.0090 \pm 0.0023$ & $<0.001$ & $-0.0058 \pm 0.0025$ & 0.019 & $-0.0077 \pm 0.0027$ & 0.005 \\
\hline
\end{tabular}

Sampling weights were applied to statistical analysis for unbiased cross-sectional estimates. $\beta$, SE, and P-values were calculated using multiple linear regression. P-values $<0.05$ are presented in bold text.

TLM, total lean mass; TFM, total fat mass; TrFM, truncal fat mass; nTrFM, non-truncal fat mass; BMD, bone mineral density; HOMA-IR, homeostasis model assessmentestimated insulin resistance; $\beta, \beta$-coefficient; SE, standard error.

${ }^{*}$ Adjusted for age, height, weight, smoking status, alcohol consumption, physical activity, household income, education, daily calcium intake, and serum vitamin D concentration. ${ }^{\dagger}$ Adjusted for HOMA-IR in addition to all variables in model $2 .{ }^{\ddagger}$ Adjusted for total cholesterol in addition to all variables in model $2 .{ }^{\S}$ Adjusted for triglyceride in addition to all variables in model 2. "Adjusted for low-density lipoprotein cholesterol in addition to all variables in model 2. 
Appendix 3. Association between TrFM, nTrFM, and BMD according to BMl subgroup

\begin{tabular}{|c|c|c|c|c|c|c|}
\hline \multirow{3}{*}{ Variable } & \multicolumn{6}{|c|}{$\mathrm{BMD}\left(\mathrm{g} / \mathrm{cm}^{2}\right)$} \\
\hline & \multicolumn{2}{|c|}{ Total femur } & \multicolumn{2}{|c|}{ Femoral neck } & \multicolumn{2}{|c|}{ Lumbar spine } \\
\hline & $\beta \pm S E$ & P-value & $\beta \pm S E$ & P-value & $\beta \pm \mathrm{SE}$ & P-value \\
\hline \multicolumn{7}{|c|}{ Obese $\left(\mathrm{BMl} \geq 25 \mathrm{~kg} / \mathrm{m}^{2}\right)$} \\
\hline \multicolumn{7}{|l|}{ Model $1^{*}$} \\
\hline TrFM & $0.0050 \pm 0.0013$ & $<0.001$ & $0.0047 \pm 0.0015$ & 0.002 & $0.0019 \pm 0.0016$ & 0.217 \\
\hline nTrFM & $0.0042 \pm 0.0017$ & 0.014 & $0.0050 \pm 0.0018$ & 0.006 & $0.0012 \pm 0.0022$ & 0.597 \\
\hline \multicolumn{7}{|l|}{ Model $2^{\dagger}$} \\
\hline TrFM & $-0.0086 \pm 0.0024$ & $<0.001$ & $-0.0068 \pm 0.0027$ & 0.013 & $-0.0077 \pm 0.0028$ & 0.006 \\
\hline nTrFM & $-0.0076 \pm 0.0021$ & $<0.001$ & $-0.0044 \pm 0.0021$ & 0.042 & $-0.0066 \pm 0.0028$ & $<0.001$ \\
\hline \multicolumn{7}{|c|}{ Non-obese $\left(\mathrm{BMl}<25 \mathrm{~kg} / \mathrm{m}^{2}\right)$} \\
\hline \multicolumn{7}{|l|}{ Model $1^{*}$} \\
\hline TrFM & $0.0098 \pm 0.0009$ & $<0.001$ & $0.0078 \pm 0.0010$ & $<0.001$ & $0.0105 \pm 0.0009$ & $<0.001$ \\
\hline nTrFM & $0.0107 \pm 0.0013$ & $<0.001$ & $0.0097 \pm 0.0014$ & $<0.001$ & $0.0103 \pm 0.0013$ & $<0.001$ \\
\hline \multicolumn{7}{|l|}{ Model $2^{\dagger}$} \\
\hline TrFM & $-0.0091 \pm 0.0014$ & $<0.001$ & $-0.0074 \pm 0.0014$ & $<0.001$ & $-0.0055 \pm 0.0014$ & $<0.001$ \\
\hline$n T r F M$ & $-0.0109 \pm 0.0016$ & $<0.001$ & $-0.0066 \pm 0.0018$ & $<0.001$ & $-0.0099 \pm 0.0017$ & $<0.001$ \\
\hline
\end{tabular}

Sampling weights were applied to statistical analysis for unbiased cross-sectional estimates. $\beta$, SE, and P-values were calculated using multiple linear regression. P-values $<0.05$ are presented in bold text.

TrFM, truncal fat mass; nTrFM, non-truncal fat mass; BMD, bone mineral density; BMl, body mass index; $\beta$, $\beta$-coefficient; SE, standard error.

*Adjusted for age, height, smoking status, alcohol consumption, physical activity, household income, education, daily calcium intake, and serum vitamin D concentration. ${ }^{\dagger}$ Adjusted for body weight in addition to all variables in model 1. 